\title{
Interference-Based Micromechanical Spectral Equalizers
}

\author{
Joseph E. Ford, Keith W. Goossen, Member, IEEE, James A. Walker, David T. Neilson, Senior Member, IEEE, \\ D. M. Tennant, Seo Yeon Park, and J. W. Sulhoff
}

\begin{abstract}
Dynamic gain equalization filters (DGEFs) are important for high-performance wavelength division multiplexed (WDM) communications. One of the first demonstrated DGEF used a micromechanical etalon filter array illuminated with free-space spectral demultiplexing optics. Here, we present subsequent research on etalon-based dynamic spectral filters, including vertical device structures which linearize and reduce the drive voltage from 70 to $40 \mathrm{~V}$, and spatially-segmented etalons which allow channelized spectral equalization and further reduce drive voltage. We describe a prototype using a simplified cylindrical optomechanical package with a 104-nm broadband spectral response, 7.5-dB insertion loss and less than 16-V operation voltage. Finally, we show the use of a 42-nm bandwidth DGEF prototype with feedback stabilization to more than double the number of channels and operating bandwidth of a conventional Erbium-doped fiber amplifier while maintaining $<1$-dB power uniformity.
\end{abstract}

Index Terms-Amplifiers, dynamic gain equalizing filters (DGEF), microelectromechanical systems (MEMS), MEMS components.

\section{INTRODUCTION}

A CTIVE power level stabilization is of great importance for high-capacity wavelength division multiplexed (WDM) transmission systems. The initial requirement was for dynamic gain equalization filters (DGEF) [1], which produce a smoothly varying spectral attenuation profile to remove variations in channel net gain profiles. The spectral resolution required corresponds to the amplifier gain variances, which are many wavelength channels wide (typically $0.8-1.6 \mathrm{~nm}$ each). A range of attenuation of $20 \mathrm{~dB}$ or more is typically needed, although specific applications can vary. The various solutions proposed and demonstrated for this application include micromechanical-based systems [2], [3], liquid-crystal [4]-[7], acousto-optic [8], and waveguide [9] technologies. A second generation of spectral equalizers with greater spectral

Manuscript received November 26, 2003; revised April 13, 2004. This research was performed at Bell Laboratories, Lucent Technologies.

J. E. Ford is with the Department of Electrical and Computer Engineering, the University of California, San Diego, La Jolla, CA 92093-0407 USA (e-mail: jeford@ucsd.edu).

K. W. Goossen is with the Department of Electrical and Computer Engineering, University of Delaware, Newark, DE 19711-3130 USA

J. A. Walker is with JayWalker Technical Consulting, Freehold, NJ 07728 USA.

D. T. Neilson and D. M. Tennant are with Bell Laboratories, Lucent Technologies, Murray Hill, NJ 07974-0636 USA.

S. Y. Park is with OptoVia Corporation, Acton, MA 01720 USA.

J. W. Sulhoff is with Onetta, Inc., Sunnyvale, CA 94089 USA.

Digital Object Identifier 10.1109/JSTQE.2004.830612 resolution was demanded in response to dynamic network reconfiguration, wherein individual wavelength channels can be switched in and out of a WDM transmission switches such as photonic cross connects and wavelength add/drop switches. Such transparent optical networking demands power level equalization of channels that have not only traversed components with nonuniform loss or gain, but may have arrived from diverse paths. Several technologies have been proposed for high resolution and dynamic range channelized equalizing filters including microelectromechanical systems (MEMS) tilt-mirror arrays [10], [11], [18], liquid crystal [5], [6], and planar light wave circuits [12].

We previously demonstrated spectral equalization using freespace optics to disperse an input spectrum over a microoptomechanical variable reflectivity etalon mirror [2]. The basic device structure, shown in Fig. 1, is a single-cavity etalon stripe with an electrically controlled air gap between a silicon substrate and a silicon nitride $\lambda / 4$ layer. Each pair of electrodes applies a local force to reduce the etalon air gap from 1.1 to $0.9 \mu \mathrm{m}$ and create smoothly varying changes in the mirror reflectivity along the length of an approximately $80 \times 1500 \mu \mathrm{m}$ optical window. Mechanical connection between adjacent regions of the membrane ensures a smoothly varying reflectivity profile.

In this paper, we present subsequent research on etalon-based dynamic spectral filters, including materials and device structures which allow linearized and reduced voltage response, spatially-segmented etalons which allow channelized spectral equalization, and a simplified and ruggedized optomechanical package. Finally, we demonstrate the use of the MEMS dynamic spectral equalizer to upgrade a conventional Erbium-doped fiber amplifier (EDFA) for increased numbers of WDM channels with wider operating parameters.

\section{OpticAl System AND OPTOMECHANICAL PACKAGE DESIGN}

The optomechanical package described in [2] used a skew optical path with vertical separation of the collimated input and output beams in the pupil to separate output signals. This package was functional, with some 4.6-dB insertion loss and $<0.2-\mathrm{dB}$ polarization dependence, but optimization required 17 separate alignments: tip, tilt, $z$ shift and rotation of the grating, tip, tilt (two controls each) and focus of two collimators, $x, y, z$, tip and tilt of the MEMS device, focus of the imaging lens, and rotation of the quarterwave plate. As such this package was practical only as a laboratory testbed. The new optical design 

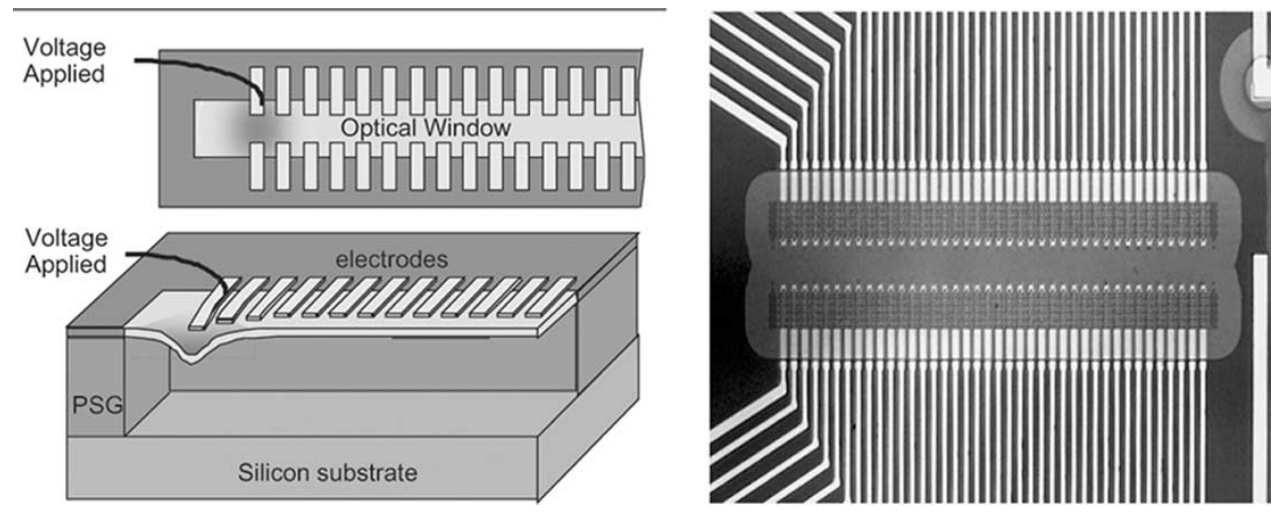

Fig. 1. MEMS micro-etalon structure (left) and fabricated device (right).

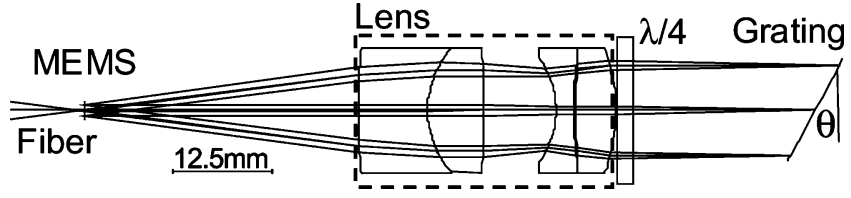

Fig. 2. Optical layout for equalizing filter, including custom double-Gauss achromatic collimation/focus lens. Distance from grating to image is $100 \mathrm{~mm}$. (Optical circulator used to separate input and output not shown.)

shown in Fig. 2 considerably simplifies optical alignment. It consists of a coaxial imaging system with a diffraction grating at the collimated beam plane and an input/output fiber located in an image plane adjacent to the MEMS device. Input and output signals are separated using an external optical circulator.

The optical demultiplexing system, illustrated in the raytrace of Fig. 2, is constructed as a folded telecentric $4 f$ system imaging system [13], [14]. The system consists of a $f=50 \mathrm{~mm}$ relay lens, with a planar $600-\mathrm{lp} / \mathrm{mm}$ grating in Littrow configuration $\left(\theta=28.3^{\circ}\right)$ at the stop. The lens is a full custom four-element achromat designed for diffraction limited resolution over a 30-nm bandwidth, as compared to the 40-nm bandwidth of the original triplet lens [3]. Light enters an input fiber and passes through an optical circulator (not shown) then is emitted from the optical fiber into free space and imaged through the diffraction grating and two passes through the lens to arrive at the MEMS device spatially dispersed by wavelength. The MEMS membrane mirror reflects the light to retrace its path through the lens and grating and be remultiplexed onto the face of the input fiber. Light coupled into the fiber propagates back to the circulator, which directs it into a separate output fiber.

This system is attractive because the full system path is $8 f$, i.e., two sequential relay imaging stages. If the MEMS device window is slightly oversized relative to the $10 \mu \mathrm{m}$ optical mode diameter, then slight lateral misalignments of the system will be compensated on the second pass and returning light will automatically realign to the fiber core. Also, since the same lens and grating is used on both passes, the multiplexing and demultiplexing angles are automatically matched. This considerably relaxes alignment and focal length tolerances.

It is essential to minimize polarization dependent loss (PDL) in the system. We selected a grating with a blaze wavelength
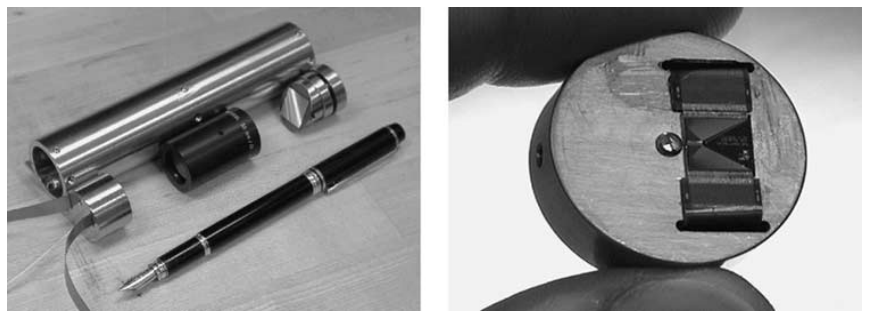

Fig. 3. (Left) Free-space WDM optomechanics, showing header, 50-mm focal length lens, and $600-1 \mathrm{p} / \mathrm{mm}$ grating. (Right) Close-up of the device, electrical flex-circuit contacts, and I/O fiber ferrule on the cylindrical metal header.

at $1.85 \mu \mathrm{m}$ wavelength with equal efficiencies (0.8-dB loss) for $S$ and $P$ polarizations at $1.55 \mu \mathrm{m}$. To eliminate small residual wavelength variance of the PDL, a zero-order quarter wave plate is placed between the lens and the grating and orientated to rotate the polarization of the light by $90^{\circ}$ upon two passes, such that the total efficiency for arbitrary input polarization light is the product of the $S$ and $P$ efficiency of the grating.

The photographs in Fig. 3 show the new optomechanical mounting, which consists of a 150-mm-long metal tube, a lens, a grating on a locking tip/tilt mount, and a cylindrical metal header. An 8-mm-square MEMS die is mounted on the 25-mm-diameter header. The 1.5-mm-long active MEMS etalon stripe is positioned next to an angle-polished ferrule, which acts as both input and output from the package. Traces on the chip are wire bonded to two identical flexible circuits, which connect 40 pairs of active leads and two grounds to the drive electronics. The system was designed to maintain the focal plane at the MEMS mirror over a $60{ }^{\circ} \mathrm{C}$ temperature range. The lens and grating mounts, tube, and header are fabricated of super-invar metal. Tests indicated a spectral shift of around $0.001 \mathrm{~nm} /{ }^{\circ} \mathrm{C}$, or about $7.5 \mathrm{GHz}$ over $60^{\circ}$. This optical system is appropriate for constructing systems operating from 1500 to $1630 \mathrm{~nm}$.

There are just four alignments, all orthogonal: the device submount slides axially for focus, the grating is tip/tilt adjusted to position the selected center wavelength on the device and rotated around the tube axis to align the dispersed spectra with the device window. The alignment process uses a broad spectrum amplified spontaneous emission (ASE) source and an optical spectrum analyzer. First, the lens and grating are inserted and 
locked in place (to an accuracy of $1 \mathrm{~mm}$ ) then the device submount is inserted and focused to peak output power. Grating tip and tilt are used to center the desired spectrum on the device window, aided by alignment marks on the die. The grating rotation is fine-tuned to align the spectral dispersion with the MEMS device window. Then, the focus, tip, and tilt are fine-tuned before locking. In this prototype, locking was done with screws; in a manufactured product, locking would be by laser welding. Alignment of the entire system requires about $5 \mathrm{~min}$, compared with approximately $4 \mathrm{~h}$ for the previous platform.

The optical performance of the system met initial design goals. The lens in an $8 f$ configuration with mirror at the back focal plane has an insertion loss of $2.4 \mathrm{~dB}$ at $1550 \mathrm{~nm}$, with a $0.2-\mathrm{dB}$ variation from 1500 to $1620 \mathrm{~nm}$. The coupling losses are those from the aberration of the optical system and from the 40 antireflection coated surfaces and the residual glass absorption. Loss could be reduced with more stringent specifications, especially on lens coatings. The grating has $1.7-\mathrm{dB}$ loss on two passes with alternate polarizations, which could also be improved. This gives a 4.1-dB insertion loss for the optical system. Two passes through the circulator adds $1.3 \mathrm{~dB}$, and the MEMS membrane $1.5 \mathrm{~dB}$, to give an expected insertion loss of $6.9 \mathrm{~dB}$. The assembled device had $7 \mathrm{~dB}$ loss, $0.2 \mathrm{ps} / \mathrm{nm}$ chromatic dispersion, and less than 0.1 ps polarization mode dispersion. The measured polarization dependent loss is less than $0.25 \mathrm{~dB}$ across the entire spectrum. The following experimental results were obtained using MEMS devices in this improved optomechanical package.

\section{LineARIZED Voltage RESPONSE MEMS DEVICE}

The mechanical anti-reflection switch (MARS) micromechanical modulator was originally developed for digital data transmission and later used as a high-speed analog variable attenuator. The basic structure is a quarter-wave dielectric antireflection coating suspended above a silicon substrate [15]. A silicon nitride layer with $1 / 4 \lambda$ optical thickness, separated from the silicon substrate by a fixed $3 / 4 \lambda$ spacer, acts as a dielectric mirror with about $70 \%(-1.5 \mathrm{~dB})$ reflectivity. Voltage applied to electrodes on top of the membrane creates an electrostatic force and pulls the membrane closer to the substrate, while membrane tension provides a linear restoring force. When the membrane gap is reduced to $\lambda / 2$, the layer becomes an antireflection coating with close to zero reflectivity. Like glass, the deformable nitride layer is brittle; the devices are defined as membranes because lateral stress is the dominant force. Mechanically, the device moves by elastic deformation, similar to a tuning fork. Electrically, the device behaves as a tiny capacitor, with zero static power dissipation, regardless of reflectivity state.

MARS device fabrication begins with deposition of a spacer layer of phospho-silicate glass (PSG) equal in thickness to the desired air gap. Next, a film of silicon nitride, with thickness set to achieve an optical path delay of one-fourth of the center operating wavelength, is deposited on the PSG layer. Both the
PSG and silicon nitride films are deposited using conventional low-pressure chemical vapor deposition (LPCVD) techniques. Electrodes, comprising a thin layer of adhesion metal (such as titanium or chrome) and a 100-nm-thick layer of gold, are deposited using a liftoff procedure. Finally, reactive-ion-etching is used to open etch access holes through the silicon nitride film exposing the PSG film. The optical window is formed and made mechanically-active by a timed hydrofluoric acid etch and rinse, followed by critical point $\mathrm{CO}_{2}$ drying.

Starting from the MARS modulator structure, a WDM equalizer can be fabricated by forming a stripe membrane lined on both sides of the optical window by mated pairs of individually-addressable electrodes, as illustrated in Fig. 1. The WDM signal is then spectrally dispersed along the length of the optical window. The $0.24-\mu \mathrm{m}$ vertical deflection required to move from maximum to minimum reflectivity is three orders of magnitude smaller than the 200 to $1500 \mu \mathrm{m}$ width and length of the membrane, making them extremely robust for literally trillions of cycles. The mechanical resonance time of such devices ranges from 0.1 to $10 \mu \mathrm{s}$ depending on the surface geometry and material parameters, particularly on membrane stress. The system response depends on drive electronics and overall packaged device capacitance.

Our initial prototype equalizer [2] used an 1150-nm-thick spacer, approximately three-fourths of the operating wavelength. The zero-voltage state is highly reflectivity $(\sim 1.5-\mathrm{dB}$ loss), decreasing to low reflectivity ( $>20$-dB loss) with approximately $70 \mathrm{~V}$ applied to a single actuator. Fig. 4(a) shows the theoretical voltage response of this device as a function of normalized bias voltage. The reflectivity depends on illuminating wavelength, so the figure plots six curves ranging from 1520 to $1620 \mathrm{~nm}$ (at 100-nm increments). Voltage response is highly nonlinear, with most of the reflectivity change occurring from $80 \%$ to $100 \%$ of applied voltage.

The $3 / 4 \lambda$-gap configuration was used so that the unpowered state of the device would be reflective (low throughput loss). However, this concern is not relevant to an equalizer used in conjunction with an amplifier, because an unpowered amplifier will absorb and block incident signals. Therefore, we can consider the alternative device shown in Fig. 4(b), where the initial gap is set at one wavelength, such that the unpowered reflectivity is relatively low This $\lambda$-gap device balances the nonlinear optical response of the optical etalon against the nonlinear voltage response of the micromechanical actuation. The reflectivity with zero bias depends strongly on wavelength, but for all wavelengths the voltage response is more linear.

The device actuation voltage depends on initial gap and the membrane stress as determined by the temperature and composition of the deposited layers. Using a silicon-rich silicon nitride layer, residual tensile stress was reduced from roughly $600 \mathrm{MPa}$ to less than $100 \mathrm{MPa}$. This reduced the actuation voltage of the new $\lambda$-gap device from 70 to $42 \mathrm{~V}$, despite the increased gap.

Fig. 5 shows the voltage response of the initial $3 / 4 \lambda$-gap device and of the low-stress $\lambda$-gap device measured at $1552 \mathrm{~nm}$. At different wavelengths, the operating voltage range is shifted. The more gradual and nearly linear change in reflectivity of 


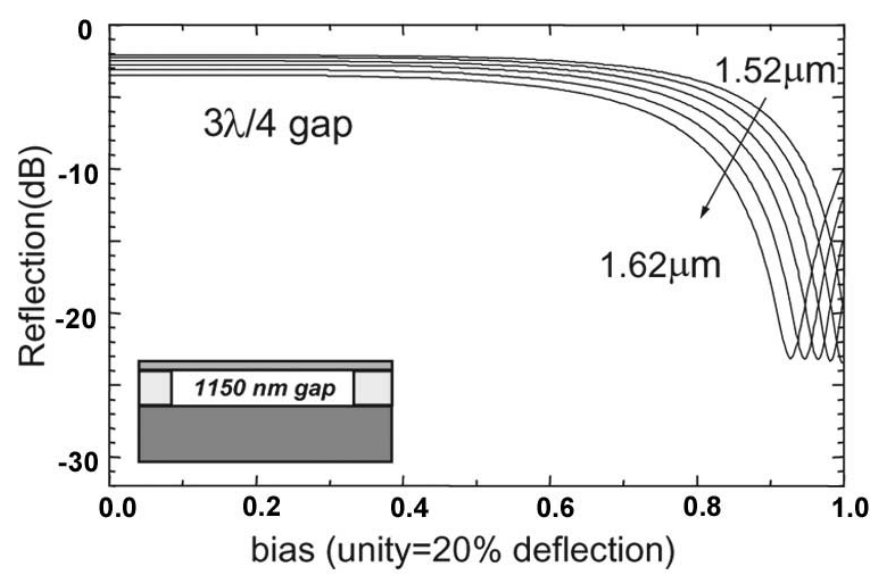

(a)

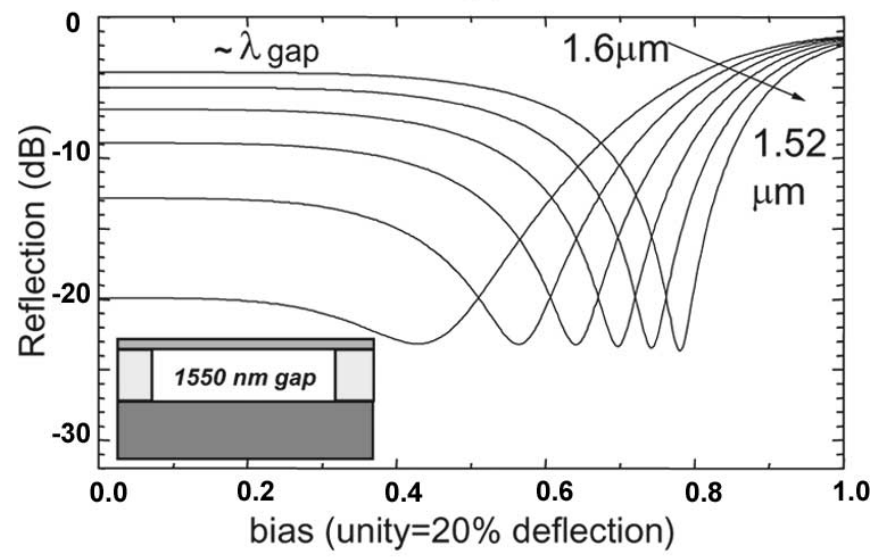

(b)

Fig. 4. Calculated microetalon reflectivity as a function of applied bias voltage for two initial air-gap spacings. (a) The initial gap is $1150 \mathrm{~nm}$, approximately $3 / 4 \lambda$. (b) The initial gap is $1550 \mathrm{~nm}$, approximately $\lambda$. In both cases, the optical delay of the nitride membrane is $\lambda / 4$.

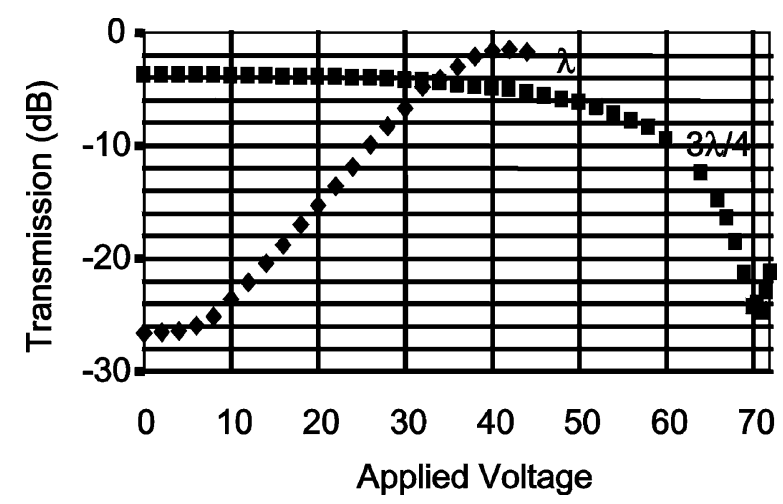

Fig. 5. Measured reflection versus applied voltage for monochromatic illumination of a $3 / 4 \lambda$ and improved $\lambda$ device, showing greater linearity in response to a reduced drive voltage.

the $\lambda$ device produces a more easily controlled response and is better suited to feedback stabilization using power monitoring.

Figs. 6 and 7 show the full spectral response of the $3 / 4 \lambda$ and $\lambda$-gap devices, respectively. Both can achieve high dynamic range $(>25 \mathrm{~dB})$ and are able to provide flat profiles, provided suitable drive voltage is applied. They also both show a 7-dB

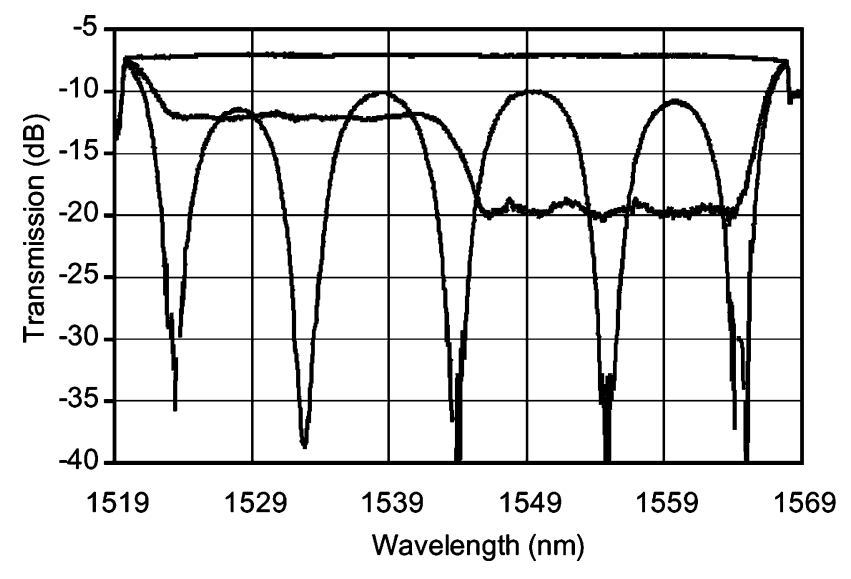

Fig. 6. Spectral response of a $3 / 4 \lambda$ device with several drive voltage settings. The top trace shows low and uniform insertion loss resulting from zero applied voltage. The trace with five deep features shows the output (and maximum dynamic range) when five single electrodes are actuated to maximum attenuation. The third trace shows the output when voltages are adjusted to achieve two uniform attenuation segments.

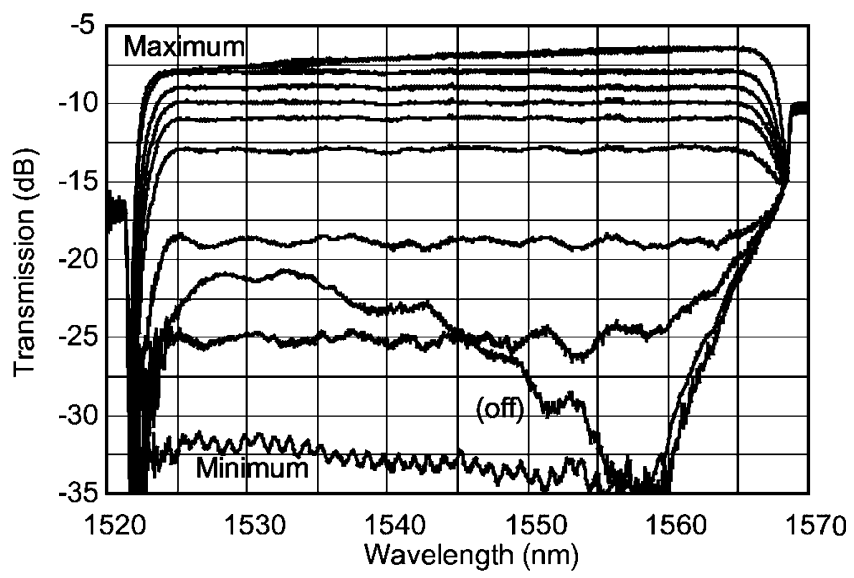

Fig. 7. Spectral response of a $\lambda$-gap device. The trace labeled "off" shows reflectivity with no applied voltage, while the trace labeled "maximum" shows output with voltages across the array set to maximize output at each wavelength. The remaining traces show the output with voltages adjusted to obtain flat response from minimum $(-32 \mathrm{~dB})$ to maximum $(-7 \mathrm{~dB})$ transmission.

insertion loss over the 42-nm design spectra. Since the $\lambda$ device has low unactuated reflectivity, it is necessary to apply a bias voltage during assembly to measure the insertion loss and achieve correct component alignment.

\section{ExTENDED $C+L$ BAND EQualizer}

A flat-spectrum white light source was needed for testing of $C+L$ band components. It was possible to build this source using two separate 42-nm equalizers. However, since both the optomechanical package and MEMS device are capable of ultra broadband performance, a $3 / 4 \lambda$ membrane equalizer with 40 actuators spaced over a 4-mm length was fabricated and mounted in the same package. The insertion loss of the resulting 104-nm bandwidth spectral equalizer is shown in Fig. 8. The minimum loss was less than $7 \mathrm{~dB}$ over the range of $1512-1600 \mathrm{~nm}$, but increased to as high as $8 \mathrm{~dB}$ at $1618 \mathrm{~nm}$. Fig. 8 also shows the attenuation created by five widely spaced features each adjusted for maximum attenuation to demonstrate 


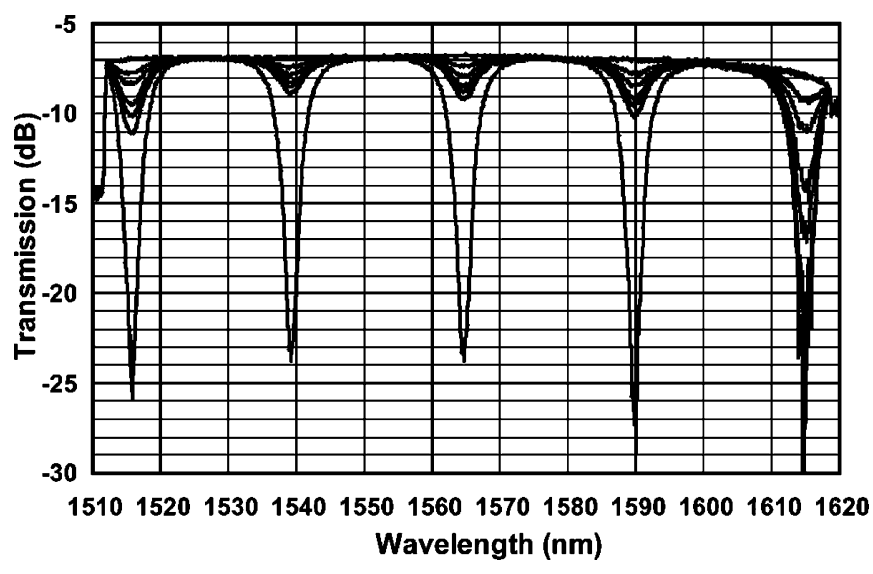

Fig. 8. Equalizer with 104-nm spectral bandwidth. Maximum drive voltage was $<16 \mathrm{~V}$, shown applied to 5 of the 40 electrodes.

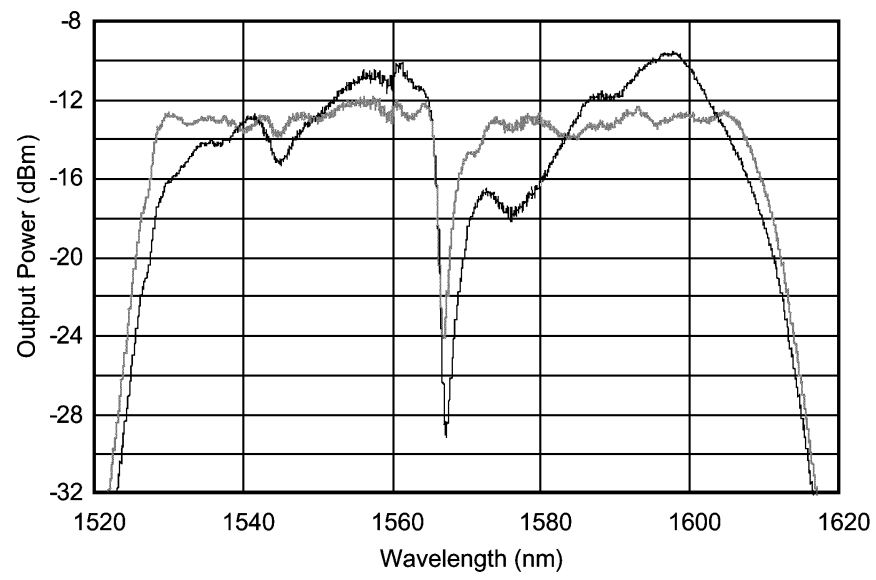

Fig. 9. Output from the same broadband spectral equalizer when used to flatten a broad spectrum $(C+L$ band) ASE source. Traces before (black) and after equalization (grey) level-flattening are shown.

that the equalizer is capable of greater than $15-\mathrm{dB}$ attenuation over the full spectral range. Low test source power limited the accuracy of measurements at the longer wavelengths, creating the noise seen in the difference spectra. The spectral features, at same attenuation levels, have the same width in the 105-nm-wide device as in the 42-nm-wide device (see the following section), indicating that the feature size results from the mechanical membrane coupling and not the optical system resolution.

Fig. 9 shows the resulting white light ASE source output before and after spectral equalization. Spectral uniformity across the $1528-1608-\mathrm{nm}$ band was improved from 9 to $+/-1 \mathrm{~dB}$, aside from the deep valley between the $C$ and $L$ bands.

The spectral width of the features could be reduced by using a longer MEMS device and a more dispersive grating system so the mechanical coupling would occur over a shorter spectral region. Compatible compact optical demultiplexing systems, with four to ten times larger spatial dispersions, have been demonstrated [10], [16], [19], which would enable spectral resolutions of $<1 \mathrm{~nm}$ to be achieved. Longer devices also require lower actuation voltages; the device illustrated in Fig. 8 required less than $16 \mathrm{~V}$ for full attenuation.

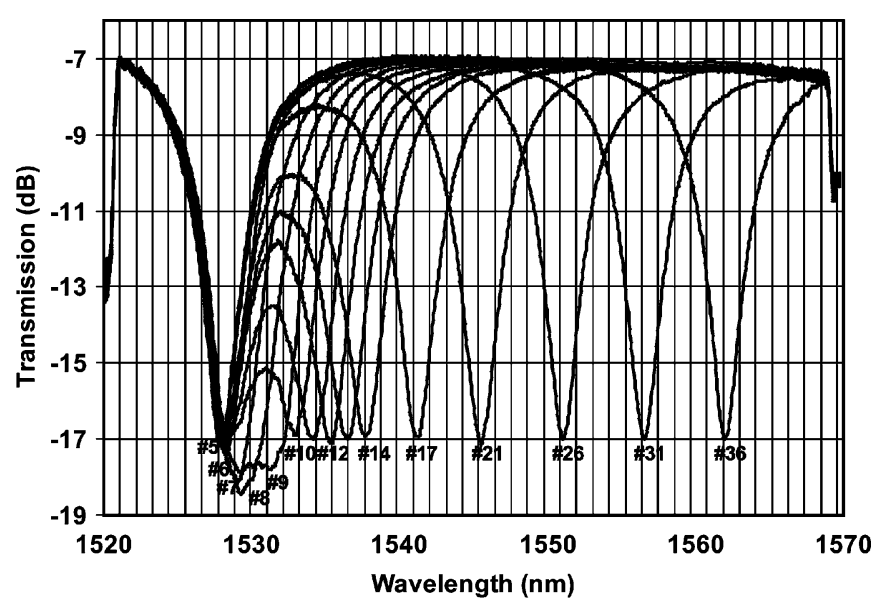

Fig. 10. Spectral resolution of the continuous membrane DGEF, showing mechanical coupling between channels that prevent discontinuous spectral profiles.

\section{Segmented “Ribbon” Channelized Equalizer}

The spectral resolution of the equalizer with a $3 / 4 \lambda$ device and a 1.5 -mm-long strip membrane with 40 actuators is illustrated in Fig. 10. One 10-dB-deep feature is created by applying voltage to a single actuator at $1528 \mathrm{~nm}$ (Channel \#5), then each trace shows the interchannel crosstalk as a second voltage is applied to successively closer actuators, each time adjusting both voltages to maintain a $10-\mathrm{dB}$ depth on the center of both features. The continuous nitride membrane has a distance-dependent mechanical cross-coupling between all actuators. For the device tested, two individual $10-\mathrm{dB}$ features create a $>1-\mathrm{dB}$ change from nominal maximum transmission midway between the features when separated by $9 \mathrm{~nm}$ and $>3 \mathrm{~dB}$ when separated by $4.5 \mathrm{~nm}$. The features can only be considered distinct when separated by over $20 \mathrm{~nm}$. The equalizer thus imposes a smoothing function on any spectral profile created, regardless of feedback. This is preferable for gain flattening filters but useless for channelized filters, where, by definition, the attenuation on each channel is independent of its neighbors. For this application, it is necessary to physically decouple the adjacent channels of the etalon equalizer.

In fact, the first conception of an etalon equalizer was based on providing one discrete equalizer device per spectral channel [2]. The equalizer stripe was composed of 32 adjacent bands, each $70 \mu \mathrm{m}$ long by $35 \mu \mathrm{m}$ wide along the spectrally dispersed direction. This equalizer was only marginally functional, as the stress in each segmented equalizer element caused the bands to curl at the edges (like potato chips). The usable passband window was less than $10 \%$ of the $200-\mathrm{GHz}$ channel spacing, making it unsuitable for practical systems. The solution for the DGEF was to use a continuous membrane, but another approach was needed for a channelized equalizer with fully independent attenuation at each wavelength data transmission channel.

One solution is to relieve the lateral stress in the membrane by slicing it into multiple thin ribbons with a common actuator. If the cuts between ribbons are smaller than the single-frequency spot size, then the cluster of ribbons still function as a variablereflectivity etalon mirror. A representative device is shown in 


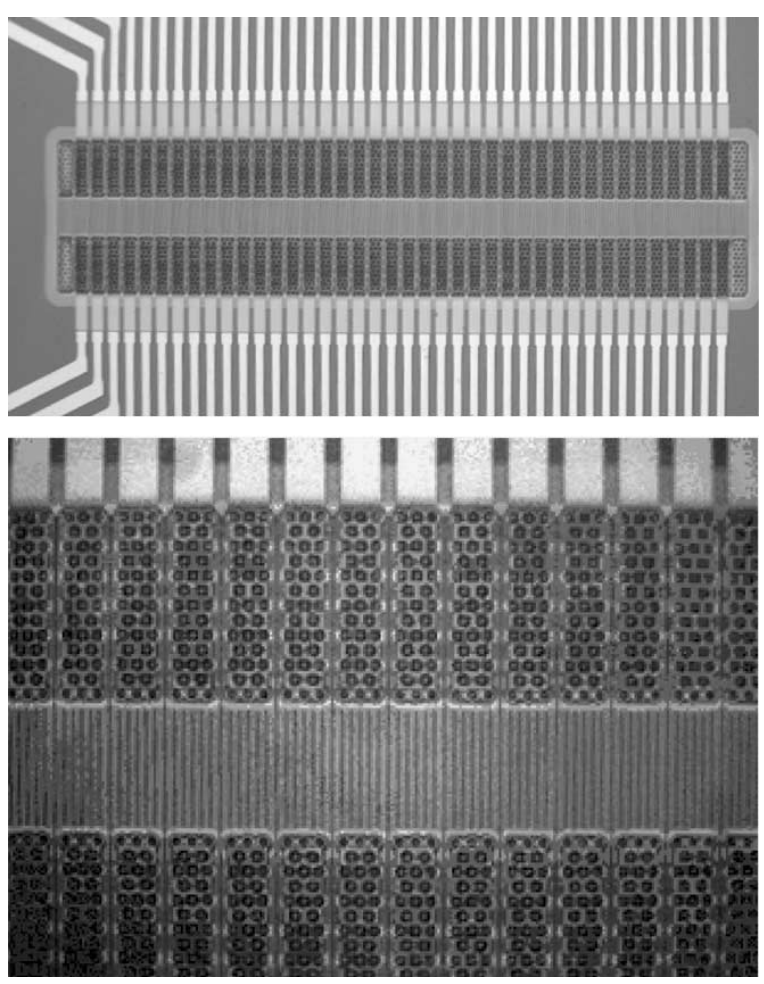

Fig. 11. Segmented equalizer device with 40 discrete mechanical actuators controlling 240 segmented ribbons plus two sets of unactuated ribbons at the ends. Top: entire device, showing 1.5-mm optical window. Bottom: closeup of segmented equalizers, showing individual ribbons.

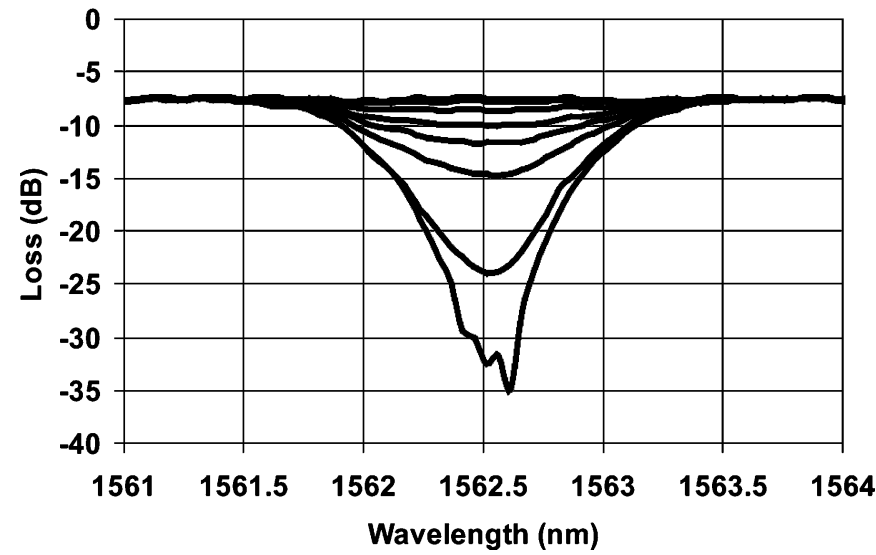

Fig. 12. Spectrum of single channel actuation at $0,5,8,10,11,12,13$, and $14 \mathrm{~V}$.

Fig. 11. As for the DGEF, the device structure is a silicon rich silicon nitride $\lambda / 4$ layer $(n=1.9)$ suspended over the silicon substrate with an electrostatically controlled gap (initially $3 \lambda / 4$ ). The individual actuators in this device were on a $36.75-\mu \mathrm{m}$ pitch, but each actuator controls a sheet of Silicon Nitride cut with submicron-width gaps into six ribbons of $6 \mu \mathrm{m}$ each. These slits were defined using e-beam lithography and etched into the Nitride using reactive ion etching.

When voltage is applied to one of the gold-coated electrode pairs, force is applied uniformly to the set of six ribbons, causing them to deflect toward the substrate and reduce etalon reflectivity from high $(-1.5 \mathrm{~dB})$ to low $(-20 \mathrm{~dB})$. The packaged device achieves an insertion loss of $8 \mathrm{~dB}$.

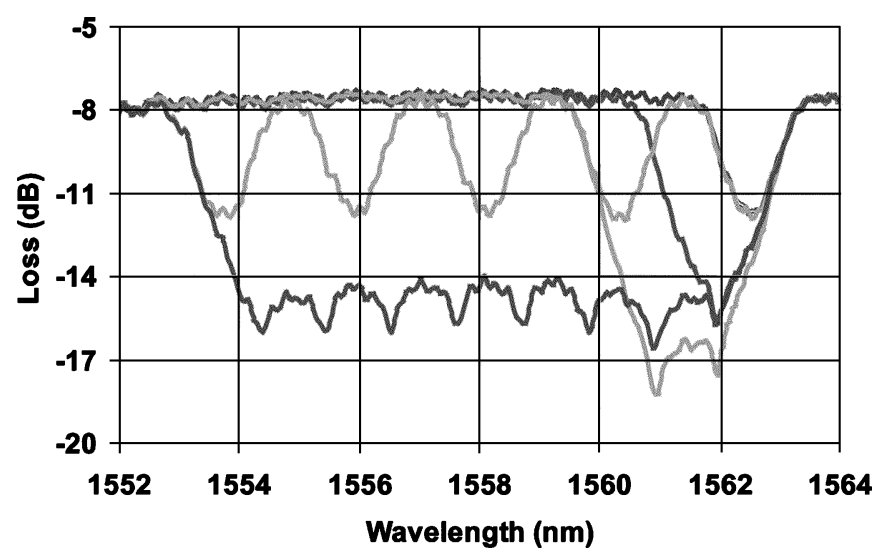

Fig. 13. Various attenuation spectra achieved by actuating from one to seven channels.

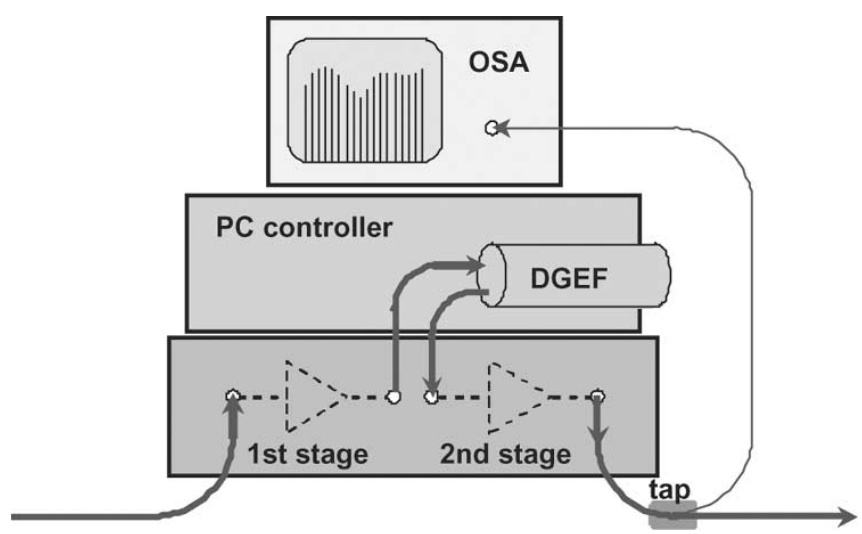

Fig. 14. Amplifier layout, with a computer-controlled dynamic gain equalization filter between two gain stages.

The system produces a spectral spacing and, hence, system resolution of $1.1 \mathrm{~nm}$ for individual actuators, compared with the spectral resolution of 5 to $10 \mathrm{~nm}$ (depending on definition) for the DGEF. Fig. 12 illustrates the response of a single actuator with 0 to $14 \mathrm{~V}$ applied, producing $20 \mathrm{~dB}$ dynamic range. Fig. 13 illustrates attenuation patterns created by actuating from one to seven different channels. The small ripples visible in the spectrum of Fig. 13 are caused by the illuminating $10.5 \mu \mathrm{m} 1 / e^{2}$ mode field diameter sampling the ribbons and spaces.

These preliminary experimental results constitute a proofof-principle for the segmented device; improving the uniformity across the passband would require further reduction of the spacing between ribbons. With physical segmentation of the actuators, creating a desired channel passband is achieved by selecting a suitable device pitch, limited only by the optical spot size illuminating the device.

\section{SElf-Equalized ERbium Amplifier}

Finally, we show how a straightforward use of the DGEF filter can dramatically enhance the operational versatility of a conventional erbium fiber amplifier. The amplifier chosen was a $C$-band EDFA originally used in the first MONET transmission system [17], designed to provide uniform gain for 16 input channels with $-8 \mathrm{dBm}$ per channel. Our goal was to increase the operating bandwidth from 12 to $30 \mathrm{~nm}$, while at the same 

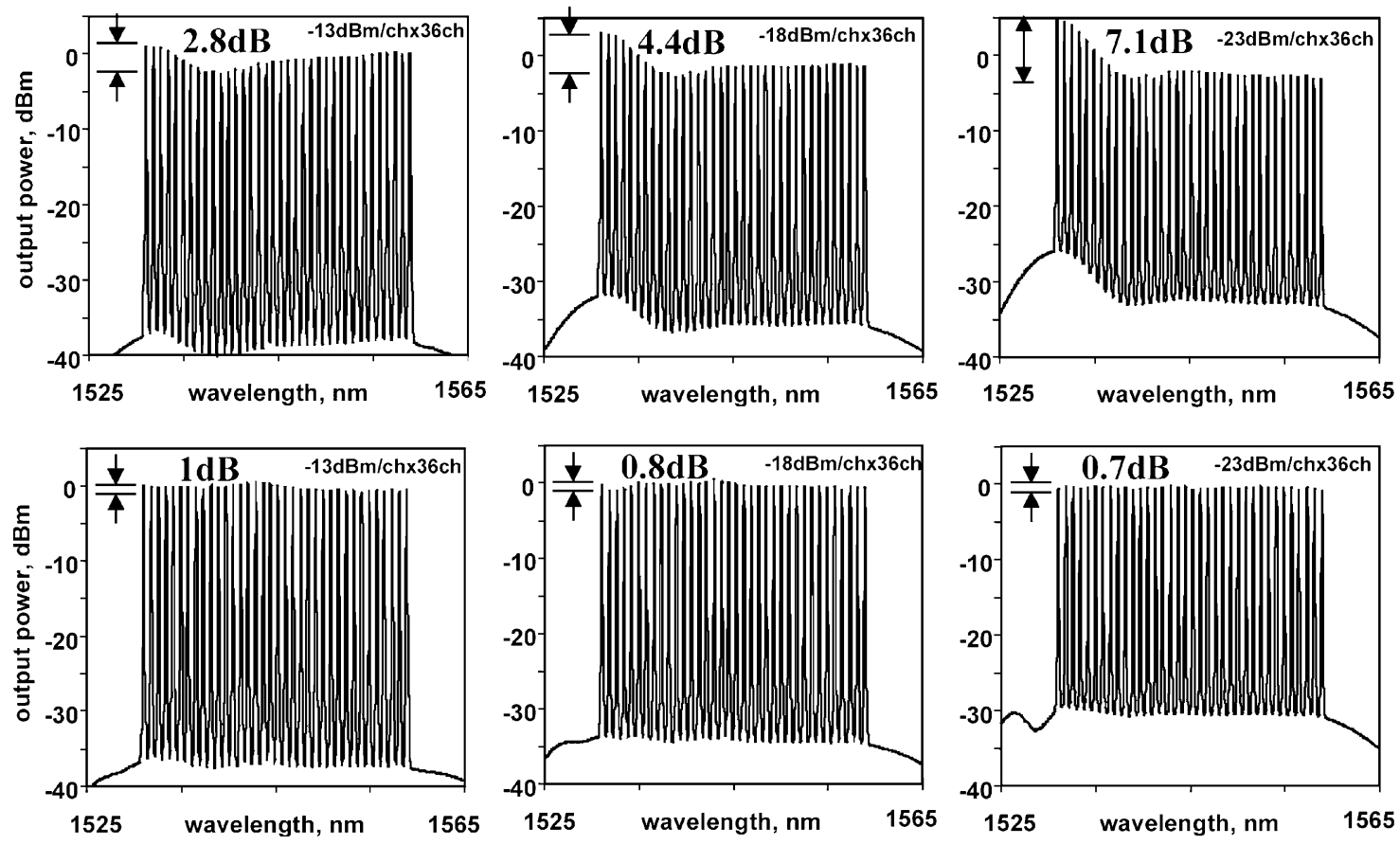

Fig. 15. Amplifier output as input power drops by 5, 10, and $15 \mathrm{~dB}$ from nominal without (top row) and with (bottom row) active spectral equalization.

time increasing the allowable input power level range to $15 \mathrm{~dB}$, all while maintaining less than $1 \mathrm{~dB}$ power nonuniformity. This capability could be used in upgrading the transmission capacity of an existing fiber span.

The original MONET amplifier provided access between two gain stages for dispersion-compensating fiber. Instead, a 42-nm-bandwidth DGEF filter was inserted between the two gain stages, as shown in Fig. 14. A 1\% output power tap was monitored by an optical spectrum analyzer connected to a PC. The PC controlled the DGEF using a simple iterative feedback stabilization algorithm incorporating a mathematical model of device voltage response.

The amplifier was tested using 36 lasers distributed over a 30-nm optical band and set at approximately equal input powers (under 1-dB level variation). The 36 signals passed through a common attenuator used to simulate loss from a variable fiber span length. The top row of traces shown in Fig. 15 show the output spectra for decreasing average input power (increasing span length) with equalization turned "off" (set to uniform maximum internal transmission). The average input power levels are $-13 \mathrm{dBm} /$ channel (top left), $-18 \mathrm{dBm} /$ channel (top center), and $-23 \mathrm{dBm} /$ channel (top right). The respective output intensities vary by up to $7 \mathrm{~dB}$ signifying poor amplifier configuration. Based on this performance for a single amplifier, the effect of cascading several such nonuniform amplifiers would be catastrophic.

However, when the equalizer is turned "on" (using feedback to create compensating attenuation profiles) the outputs converge to $1 \mathrm{~dB}$ or lower variation for all cases, as seen in the bottom row of traces in Fig. 15. In this case, the feedback signal is local to the DGEF, but it is possible to remotely monitor the optical power levels and use this signal to control a single equalizer at midspan, thereby providing end-to-end equalization. In
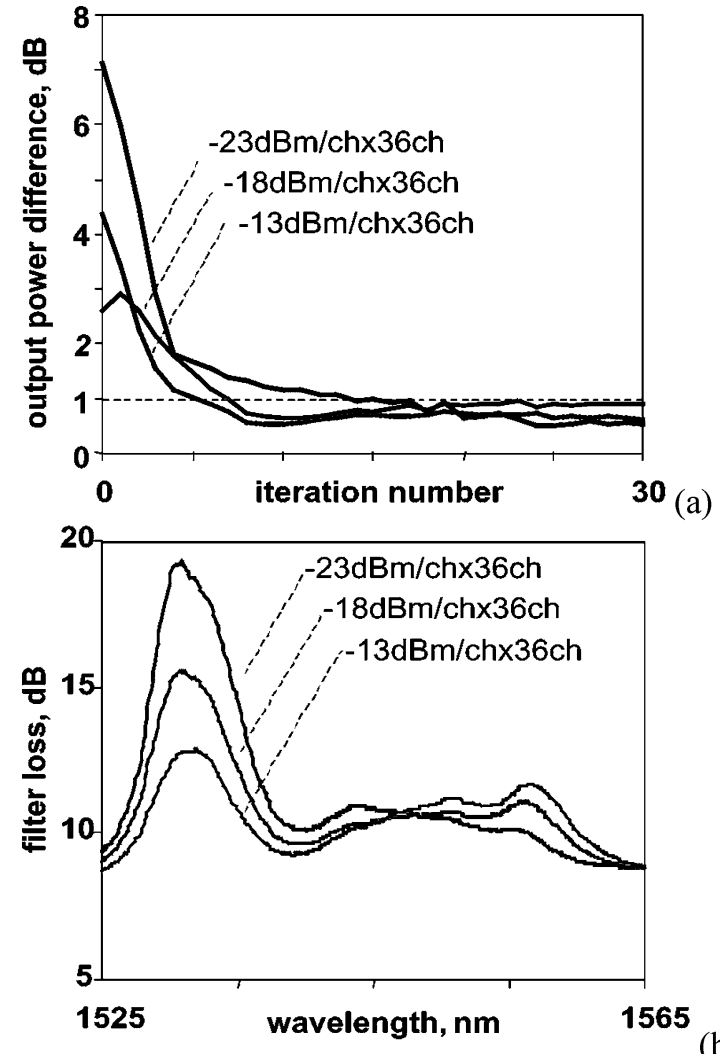

(b)

Fig. 16. (a) Output power variation as a function of time after a sudden change in input power level. (b) Resulting gain equalization filter profiles.

practice, the number of equalizers needed would depend on the allowed mid-span power divergence before an unacceptable level of nonlinear transmission impairment occurred.

Fig. 16(a) shows output power convergence as a function of time following a sudden drop in input intensity. Each feedback 
loop iteration required about $0.5 \mathrm{~s}$ for the optical spectrum analyzer to collect and transfer the spectrum, but using a fast power monitor would reduce this to less than $100 \mathrm{~ms}$.

Fig. 16(b) shows the steady state attenuation profiles of the equalization filter, from which the compensation of the gain tilt is apparent. The filter has a uniform minimum insertion loss of about $7 \mathrm{~dB}$, and applies an additional loss of up to $10 \mathrm{~dB}$ as the input power decreases. Since the amplifier is operated in a gainsaturated regime, the resulting output power is not significantly affected. The noise figure (indicated by the ASE level between signals) does show some increase, but maintains a signal to ASE difference of $30 \mathrm{~dB}$ or more.

\section{CONCLUSION}

Dynamic channel equalization may be implemented with various device technologies or even combined with other network functions such as channel monitors and transparent switching. Clearly, dynamic spectral filters can be constructed using freespace or waveguide demultiplexing in combination with a variety of actuator technologies. In this paper, we have shown a range of equalizers based on micromechanical etalons, including device structures for reduced drive voltage, more linear response, and great spectral resolution. We have also demonstrated the utility of dynamic gain equalization filters for creating more versatile amplifiers and, by extension, WDM transmission systems. We believe that dynamic channel equalization, however it is implemented, is well on its way to becoming an essential function in WDM networks.

\section{REFERENCES}

[1] W. J. Tomlinson, "Dynamic gain equalization for next-generation DWDM transport systems," in Dig. LEOS Summer Topical Meet. Ultralong Haul DWDM Transmission Networking/WDM Components, July 30-Aug. 1 2001, p. 2.

[2] J. E. Ford, J. A. Walker, M. C. Nuss, and D. A. B. Miller, "32-channel WDM graphic equalizer," in Dig. IEEE/LEOS Summer Topical Meet. Broadband Optical Networks, Keystone, CO, 1996, pp. 26-27.

[3] J. E. Ford and J. A. Walker, "Dynamic spectral power equalization using micro-opto-mechanics," IEEE Photon. Technol. Lett., vol. 10, pp. $1440-1442$, Oct. 1998.

[4] A. Ranalli, B. Scott, and J. Kondis, "Liquid crystal—based wavelength selectable cross-connect," in Proc. Eur. Conf. Optical Communications, Nice, France, 1999, pp. 68-69.

[5] J. K. Rhee, F. Garcia, A. Ellis, B. Hallock, T. Kennedy, T. Lackey, R. G. Lindquist, J. P. Kondis, B. A. Scott, J. M. Harris, D. Wolf, and M. Dugan, "Variable passband optical add-drop multiplexer using wavelength selective switch," in Proc. 27th Eur. Conf. Optical Communication, vol. 4, 2001, pp. 550-551.

[6] O. Bouevitch, D. Touahri, J. P. Morgan, S. Panteleev, and C. Reimer, "Channel-power equalizer and dynamic gain equalizer based on the optical bench platform," in Dig. IEEE/LEOS Summer Topical Meet. AllOptical Networking: Existing and Emerging Architecture and Applications/Dynamic Enablers of Next-Generation Optical Communications Systems/Fast Optical Processing in Optical Transmission/VCSEL and Microcavity Lasers, July 15-17, 2002, pp. MD1-4-MD1-5.

[7] J.-C. Chiao, "Liquid-crystal optical harmonic equalizers," in Dig. LEOS Summer Topical Meet. Advanced Semiconductor Lasers and Applications/Ultraviolet and Blue Lasers and Their Applications/Ultralong Haul DWDM Transmission and Networking/WDM Components, July 30-Aug. 1 2001, pp. 39-40.
[8] H. S. Kim, S. H. Yun, H. K. Kim, N. Park, and B. Y. Kim, "Dynamic gain equalization of erbium-doped fiber amplifier with all-fiber acoustooptic tunable filters," in Tech. Dig. Optical Fiber Communication Conf. Exhibit, Feb., 22-27 1998, pp. 136-138.

[9] C. R. Doerr, C. H. Joyner, and L. W. Stulz, "Integrated WDM dynamic power equalizer with potentially low insertion loss," IEEE Photon. Technol. Lett., vol. 10, pp. 1443-1445, Oct. 1998.

[10] D. T. Neilson, D. S. Greywall, S. Chandrasekhar, L. L. Buhl, H. Tang, L. Ko, N. R. Basavanhally, F. Pardo, D. A. Ramsey, J. D. Weld, Y. L. Low, J. Prybyla, R. Scotti, A. Gasparyan, M. Haueis, S. Arney, S. P. O’Neill, C.-S. Pai, D. H. Malkani, M. M. Meyers, N. Saluzzi, S. H. Oh, O. D. Lopez, G. R. Bogart, F. P. Klemens, M. Luo, J. Q. Liu, K. Teffeau, A. Ramirez, K. S. Werder, J. E. Griffith, C. Frye, M. V. Kunnavakkam, S. T. Stanton, J. A. Liddle, H. T. Soh, T.-C. Lee, O. Nalamasu, and K. C. Nguyen, "High-dynamic range channelized MEMS equalizing filter," in Proc. Optical Fiber Communication Conf. Exhibit, 2002, pp. 586-588.

[11] D. M. Marom, D. T. Neilson, D. S. Greywall, V. Aksyuk, M. E. Simon, N. R. Basavanhally, P. R. Kolodner, Y. L. Low, F. Pardo, C. A. Bolle, C. S. Pai, D. López, J. A. Taylor, J. E. Bower, J. Leuthold, M. Gibbons, and C. R. Giles, "Wavelength selective $4 \times 1$ switch with high spectral efficiency, $10 \mathrm{~dB}$ dynamic equalization range and internal blocking capability," in Proc. Eur. Conf. Optical Communications, Rimini, Italy, Sept. 21-25, 2003, Paper No 3.5.3.

[12] C. R. Doerr, L. W. Stulz, R. Pafchek, and S. Shunk, "Compact and low-loss manner of waveguide grating router passband flattening and demonstration in a 64-channel blocker/multiplexer," IEEE Photon. Technol. Lett., vol. 14, pp. 56-58, Jan. 2002

[13] J. E. Ford, J. A. Walker, K. Goossen, and D. T. Neilson, "Broad spectrum micromechanical equalizer," in Proc. Eur. Conf. Optical Communications, Nice, France, Sept. 26, 1999.

[14] J. E. Ford, "Optomechanical platform," U.S. Patent 6307 657, Oct. 23, 2001.

[15] J. E. Ford, J. A. Walker, D. S. Greywall, and K. W. Goossen, "Micromechanical fiber-optic attenuator with 3 microsecond response," IEEE J. Lightwave Technol., vol. 16, pp. 1663-1670, Sept. 1998

[16] D. T. Neilson, R. Ryf, F. Pardo, V. A. Aksyuk, M.-E. Simon, D. O. Lopez, D. M. Marom, and S. Chandrasekhar, "MEMS based channelized dispersion compensator with flat passbands," IEEE J. Lightwave Technol., vol. 22, pp. 101-105, Jan. 2004.

[17] W. T. Anderson, J. Jackel, G.-K. Chang, H. Dai, W. Xin, M. Goodman, C. Allyn, M. Alvarez, O. Clarke, A. Gottlieb, F. Kleytman, J. Morreale, V. Nichols, A. Tzathas, R. Vora, L. Mercer, H. Dardy, E. Renaud, L. Williard, J. Perreault, R. McFarland, and T. Gibbons, "The MONET project-A final report," IEEE J. Lightwave Technol., vol. 18, pp. 1988-2009, Dec. 2000

[18] T. Giles, "Wavelength selective $1 \times 4$ switch for 128 WDM channels at $50 \mathrm{GHz}$ spacing," in Proc. Optical Fiber Communications Conf. Exhibit, 2002, pp. 857-859.

[19] D. T. Neilson, R. Ryf, F. Pardo, V. A. Aksyuk, M.-E. Simon, D. O. Lopez, D. M. Marom, and S. Chandrasekhar, IEEE J. Lightwave Technol., vol. 22, pp. 101-105, Jan. 2004.

Joseph E. Ford received the B.Sc. degree in physics from the University of California, Los Angeles, in 1983, the M.S. degree in laser physics from the University of British Columbia, Vancouver, BC, Canada, in 1985, the M.S. degree in optical engineering from the Institute of Optics, University of Rochester, Rochester, NY, in 1986, and the Ph.D. degree in electrical engineering/applied physics from the University of California at San Diego (UCSD), La Jolla, CA, in 1992.

From 1994 to 2000, he was with the Advanced Photonics Research Department, Lucent Bell Laboratories, Holmdel, NJ, where he worked on integrating surface-normal optoelectronic and micromechanical devices with single-mode fiber for optical modulation, switching, and data transmission. From 2000 to 2002, he was with Optical Micro-Machines (OMM), San Diego, CA, an optical switching startup. In 2002, he became an Associate Professor with the Department of Electrical and Computer Engineering, UCSD, where he started the Photonics Systems Integration Laboratory. 
Keith W. Goossen (S'87-M'88) received the B.S. degree from the University of California at Santa Barbara, and the Ph.D. degree from Princeton University, Princeton, NJ, both in electrical engineering.

After receiving his degrees, he became a Member of Technical Staff at Bell Laboratories, first as part of AT\&T, then Lucent Technologies, Holmdel, NJ. While there, he invented and demonstrated the world fastest MEMS optica modulator, which was used in system demonstrations for ultra-broadband fiber-to-the-home networks and dynamic wavelength management in wavelength division multiplexed networks. He also invented and demonstrated flip-chip bonding techniques for integrating electronic and photonic chips, particularly short-wavelength vertical cavity surface emitting laser (VCSEL) chips, which resulted in the first demonstration of optical transceiver chips capable of transmitting, receiving, and processing up to a terabit/second of data. In 2000, he co-founded Aralight, Inc., Jamesburg, NJ, as a Chief Scientist, to commercialize this technology for optical backplane applications, and guided the engineering development which resulted in a full product demonstration two years later of 36-channel, $90-\mathrm{Gb} / \mathrm{s}$ transmit/receive parallel optical modules. After reaching this prototype stage, he joined the University of Delaware, Newark, to re-engage in the pursuit of technology research, including extending dense parallel optical device technology to broad computing impact, exploring the per channel data rate limitations of VCSELs and developing external modulator technologies to surpass those limitations, furthering the performance and application range of MEMS optoelectronics, and using the principles of optical fiber communication to develop metropolitan wide sensor net technology for advanced societal impact and benefit of metrology. $\mathrm{He}$ is currently an Associate Professor with the Department of Electrical and Computer Engineering, University of Delaware. He has 15 years professional experience in the fields of optoelectronics and optical fiber communication, which has resulted in over 100 refereed journal papers, numerous invited talks and conference proceedings, and 73 patents.

James A. Walker received the B.S. and M.S. degrees in electrical engineering from Rutgers University, New Brunswick, NJ, in 1984 and 1989, respectively.

He joined Bell Laboratories, Lucent Technologies, Holmdel, NJ, in 1984 and was a Member of Technical Staff and Technical Manager of the MEMS Network Element Subsystems Group until June 2000. He subsequently spent two years as the Director of Advanced Technologies at Tellium, Inc., Oceanport, NJ, where he jointly led an effort to develop all-optical MEMS-based cross-connect switching systems. He now heads a team of MEMS and telecom consultants as the President of JayWalker Technical Consulting, LLC, Freehold, NJ. He is also a Registered Patent Agent with the U.S. Patent and Trademark Office, and provides patent prosecution expertise in the areas of MEMS, nanotechnology, optical telecom, and opto-mechanical packaging. He holds 53 U.S. patents, has authored over 100 journal articles and conference publications, presented numerous invited and submitted conference papers in the areas of photonics and MEMS, and chaired several international conferences in the area of optical MEMS technology.
David T. Neilson (M'96-SM'02) received the B.Sc. degree (with honors) in physics and the Ph.D. degree in physics for work on optical nonlinearities in InGaAs quantum-well devices from Heriot-Watt University, Edinburgh, U.K., in 1990 and 1993, respectively.

He was a Postdoctoral Researcher with Heriot-Watt University from 1993 to 1996, where he was working with systems and devices for free-space optical interconnects. From 1996 to 1998, he was a Visiting Scientist at NEC Research Institute, Princeton, NJ, where he was researching optical interconnects for high-performance computing. In 1998, he joined Bell Laboratories, Lucent Technologies, Murray Hill, NJ, where he has worked on MEMS-based crossconnects, wavelength selective switches, equalizers, and dispersion compensators, and where he is currently a Technical Manager with responsibility for optoelectronic device growth and fabrication facility. He has over 80 publications in the field of optical interconnects and switching.

Dr. Neilson is a senior member of the IEEE Lasers and Electro-Optics Society and a member of the Optical Society of America.

D. M. Tennant, photograph and biography not available at the time of publication.

Seo Yeon Park received the B.S. and M.S. degrees in electronics engineering from Yonsei University, Seoul, Korea, in 1987 and 1989, respectively, and the $\mathrm{Ph} . \mathrm{D}$. degree in electrical engineering from the Korea Advanced Institute of Science and Technology, Taejon, Korea, in 1998.

Between 1989 and 1998, he was with the Electronics and Telecommunications Research Institute, Taejon, as a Research Engineer. From 1999 to 2000 he was a Member of Technical Staff at Bell Laboratories, Advanced Optical Networking Center, Lucent Technologies, Holmdel, NJ. From 2000 to 2002, he was with Sycamore Networks, Chelmsford, MA, as a Principal System Engineer. In 2003, he joined Optovia Corporation, Acton, MA, as an optics design lead. His research interests include optical amplifiers, optical fiber communications, and WDM optical networkings.

J. W. Sulhoff, photograph and biography not available at the time of publication. 